\title{
Cloud-based framework to mitigate the impact of COVID-19 on seafarers' mental health
}

\author{
Mamta Mittal ${ }^{1} \oplus$, Gopi Battineni ${ }^{2} \oplus$, Lalit Mohan Goyal ${ }^{3} \oplus$, Bijoy Chhetri ${ }^{3}$, \\ Sonia Vashishta Oberoi ${ }^{4}$, Nalini Chintalapudi ${ }^{2}{ }^{\infty}$, Francesco Amenta ${ }^{2,5}$ \\ ${ }^{1}$ Department of CSE, G B Pant Government Engineering College, Okhla, New Delhi, India \\ ${ }^{2}$ Telemedicine and Telepharmacy Centre, School of Medical Products and Sciences, University of Camerino, Italy \\ ${ }^{3}$ Department of CE, J C Bose University of Science and Technology, YMCA, Faridabad, India \\ ${ }^{4}$ Former Professor, Department of Arts and Humanities, REVA University Bangalore, India \\ ${ }^{5}$ Research Department, Centro Internazionale Radio Medico (C.I.R.M.), Rome, Italy
}

Today the entire world is flabbergasted by the sheer magnanimity of the novel corona virus disease (COVID-19). Its infection and death rates are unprecedented and are manifolds as compared to the other fatal epidemics of history [1]. This contagious disease has affected people worldwide without any discrimination of class, colour, or gender. Though the facts suggest that normalcy will return, yet the terrifying blemishes of COVID-19 will be there for a very long time on the human mind. Much psychological distress will prevail across the world.

It can also be observed that there is an instance of psychological imbalance among people working on enclosed environments such as seafarers. At this rate of infection and death worldwide, challenges are emerging to keep everyone psychologically well. Psychiatrist intervention is much required today [2]. To lessen the psychological vulnerabilities among ship workers, it is time to understand the science behind the disease rather than fearing it. Psychiatrists, psychiatric nurses, clinical psychologists, and other mental health workers should bridge the gap between fear and reality [3]. It is quite evident that there is an inherent characteristic of epidemics including COVID-19 to have comorbidity with psychological disorders related to either fear or financial distress. There are also onboard-infected people, and their caretakers in the affected area who are vulnerable to psychological disorders.

When someone gets mild symptomatic in ship environments, a greater risk of fear and stress would make that person more vulnerable. This vulnerability causes various psychological problems like distress, depression, anxiety, insomnia and even suicidal death. Online assessment to understand emotional and behaviour fear assessment can be made available to ease out stress, depression, and fear [4]. Various computational and learning algorithms need to be put in place to preempt the psychological imbalance like the risk of suicide, lessen human manoeuvrability within the affected marine environments, prediction of viral relapse and classification and containment of the disease, etc. which would amend the calibre of effectiveness during emergency interventions.

Thus, a cloud-based technology has been designed to assist seafarers from onshore by proactively managing psychiatric concerns like depression, anxiety, and other conditions with the employment of predictive analytics powered by an Artificial Intelligence models. The main contribution of this work is to present a conceptual framework for the monitoring of mental conditions of COVID-19-infected seafarers. This framework is presented in Figure 1, to support the clinical care, psychiatric issue assessment, educating onboard staff, and online self-assessment of individuals as well as depressed people. It can also help to control psychiatric issues utilising individual communications with other seafarers through simple chat sessions.

This framework model has been designed with the integration of both cloud and artificial intelligence technologies. The database of incident management system collects the local data of mental and physical states of onboard isolated (or quarantined) seafarers by COVID-19. The seafarer can be registered into the given application and record the parameters (i.e., body temperature, pulse rate, body mass index, blood pressure, heart rate, etc.) to monitor regular health conditions. When an onboard person feels that he/she got a sudden hike in body temperature, or having breathing issues, smart 


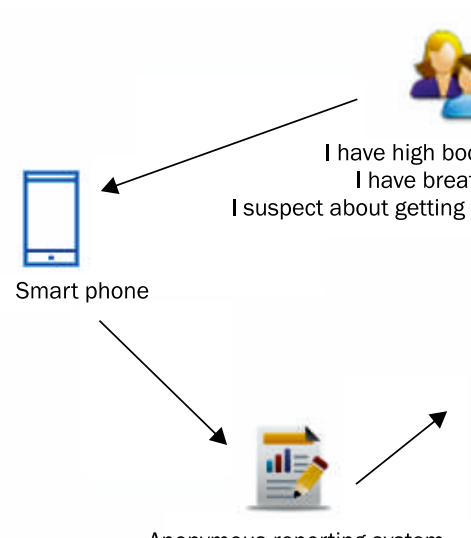

Anonymous reporting system

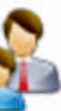

dy temparutre

Discharge yesterday Are you alone

Hows your health now
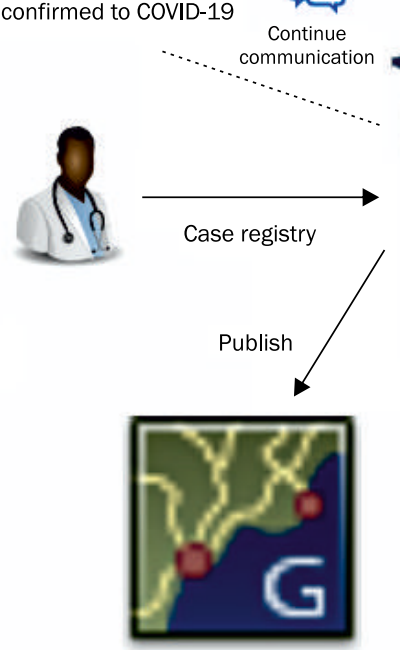

COVID-19 tracker.org </my town>

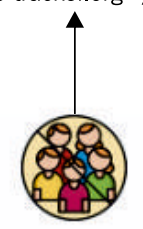

I want to know how many people
are infected in my town

Is it possible to go to markets

Figure 1. Cloud-based framework to monitor the mental conditions of COVID-19 infected patients

phone application alerts the seafarer and makes an immediate appointment by contacting the emergency centre. The incident management system always records the number of infected people in a particular ship and shares the daily effective infection rates with ship authorities. Thanks to publishing or sharing experiences of recovery patients enable infected onboard person can feel better and more confident to fight against COVID-19. In this way, the proposed framework can open new doors to use seafarer's safety technology to save lives during this new pandemic by providing a comprehensive way of track and manage individual's COVID-19 responses.

The supplementary material associated with artificial intelligence chatbot application during the COVID-19 pandemic can be found at: https://doi.org/10.3390/healthcare8020154.

\section{ACKNOWLEDEGEMENTS}

This paper was supported in part by the ITF Trust grant No. 1508/2020 to Centro Internazionale Radio Medico (C.I.R.M.).

\section{REFERENCES}

1. Yang HY, Duan GC. Analysis on the epidemic factors for the corona virus disease. Zhonghua Yu Fang Yi Xue Za Zhi. 2020; 54(0): E021, doi: 10.3760/cma.j.cn112150-20200227-00196.

2. Banerjee D. The COVID-19 outbreak: Crucial role the psychiatrists can play. Asian J Psychiatry. 2020; 50: 102014, doi: 10.1016/j. ajp.2020.102014.

3. Shi Y, Wang J, Yang Y, et al. Knowledge and attitudes of medical staff in Chinese psychiatric hospitals regarding COVID-19. Brain Behav Immun Health. 2020; 4: 100064, doi: 10.1016/j.bbih.2020.100064, indexed in Pubmed: 32289123.

4. Ahorsu D, Lin CY, Imani V, et al. The Fear of COVID-19 Scale: deveIopment and initial validation. Int J Mental Health Addiction. 2020, doi: 10.1007/s11469-020-00270-8. 